\title{
O surgimento dos Observatórios de Cultura e de Políticas Culturais: Reflexões inicias para construção de tipologias
}

\author{
El surgimiento de los Observatorios de la Cultura y Políticas \\ Culturales: Reflexiones iniciales para la construcción de tipologías
}
The emergence of the Culture and Cultural Policies Observatories: Initial reflections for typologies construction

\author{
Clarissa Semensato ${ }^{1}$
}

\begin{abstract}
Resumo:
Palabras chave:

O surgimento dos observatórios de cultura e políticas culturais é um fenômeno recente, e cada vez mais tem crescido o número desse tipo

Observatórios de Cultura de organismo. Apesar desse fato, é escassa a bibliografia a respeito dos Observatórios de Cultura e Políticas Culturais. Algumas poucas

Políticas Culturais

América Latina informações já foram levantadas a respeito daqueles existentes na Europa, porém, pesquisas sobre esta temática na América Latina ainda são carentes, a despeito do fortalecimento do fenômeno nessa região. Na iniciativa de amenizar a carência de informações, este estudo é uma pesquisa exploratória sobre os Observatórios de Cultura e Políticas Culturais existentes na região. O objetivo deste artigo é mapear os observatórios que se propõem a cobrir a temática cultura e políticas culturais, existentes no Brasil, Chile, Argentina e Colômbia, com foco no Brasil. Através da identificação do que eles declaram como responsabilidades e missão, bem como suas fontes de financiamento, pretende-se colaborar com a construção de tipologias. Estas, por sua vez, devem fomentar reflexões que darão base para a criação de novos observatórios, bem como para reflexões sobre o fazer daqueles já existentes.
\end{abstract}




\section{Resumen:}

El surgimiento de los observatorios de la cultura y políticas culturales es un fenómeno reciente, y cada vez más se ha incrementado el número de dichos órganos. A pesar de eso, es escasa la literatura sobre los Observatorios de Cultura y Políticas Culturales. Pocos detalles se han planteado en relación a los observatorios existentes en Europa, sin embargo, la investigación sobre este tema en América Latina siguen deficiente, a pesar del fortalecimiento del fenómeno en esta región. Con la iniciativa de reducir la falta de información, este estudio es una investigación exploratoria sobre los Observatorios de la Cultura y Políticas Culturales existentes en la Latinoamérica. El propósito de este artículo es mapear los observatorios que se proponen cubrir el tema de la cultura y políticas culturales existentes en Brasil, Chile, Argentina y Colombia, con un enfoque en Brasil. Mediante la identificación de lo que se declara como sus responsabilidades y misión, así como sus fuentes de financiamiento, pretenda se colaborar con la construcción de tipologías. Estas, a su vez, deben estimular reflexiones que dan base para la creación de nuevos observatorios, así como reflexiones sobre la práctica de aquellos existentes.

\section{Palabras clave:}

Observatorios de Cultura

Políticas Culturales

Latinoamérica

\section{Keywords:}

\section{Culture Observatories}

Cultural Policies

Latin America

\section{Abstract:}

The emergence of culture and cultural policies observatories is a recent phenomenon, and progressively the number of such bodies has increased. Despite the fact, the literature about Culture and Cultural Policies Observatories is scarce. Some information concerning those existing observatories in Europe are available, however, sources regarding this topic in Latin America are still deficient, despite the strengthening of this phenomenon in the region. In an effort to mitigate the lack of information, this study is an exploratory research of the existing Culture and Cultural Policies Culture Observatories in Latin America. The purpose of this article is to map the observatories that are dedicated to cover the existing culture and cultural policies in Brazil, Chile, Argentina and Colombia, with a focus on Brazil. By identifying what they have established as their responsibilities and mission, as well as their financial sources, we intend to collaborate by building typologies. These, in turn, should encourage reflections that give basis for the creation of new observatories, as well as considerations on practices of the existing ones. 


\section{O surgimento dos Observatórios de Cultura e de Políticas Culturais: reflexões inicias para construção de tipologias}

\subsection{O fenômeno dos Observatórios de Cultura}

Ao final do século $X X$ começa a ser constatado o fenômeno do surgimento dos Observatórios em vários lugares do mundo. Nos últimos anos, provavelmente devido à valorização de políticas culturais, constata-se uma proliferação de instituições tipo observatório com a temática da cultura. Assim, os Observatórios de Cultura, especificamente, são fenômeno bastante recente, afinal a prática de coletar, sistematizar e analisar de dados específicos sobre a cultura faz parte de uma preocupação nascida a apenas duas ou três décadas em algumas nações altamente industrializadas, e há menos de uma década nos países da América Latina (GETINO, 2007, p. 44).

Há poucos estudos acadêmicos específicos sobre o fenômeno dos Observatórios. São ainda mais escassos aqueles que têm como recorte a América Latina. $\mathrm{Na}$ intenção de colaborar com a produção acadêmica que visa refletir sobre o crescimento desse número e o papel dos observatórios de cultura, este artigo se propõe a mapear os observatórios de cultura e políticas culturais, ativos do Brasil, Chile, Argentina e Colômbia, com ênfase no Brasil. Através da identificação do que eles declaram como missão, sua atuação, fontes de financiamento e raio de atuação, pretende-se dar apontamentos para construção de tipologias. A definição de tipologias tem o potencial de fomentar pesquisas e reflexões que podem embasar a criação de novos observatórios, ou ainda fomentar reflexões sobre as atividades daqueles já existentes.

A Organização de Estados Iberoamericanos (OEI) concluiu que o surgimento dos observatórios de cultura ocorre num primeiro momento, na Europa, mas logo a prática se estende a outros continentes. Eles nascem da necessidade de sistematizar as fontes de informações e realizar análises sistemática das Políticas Culturais, pesquisa e planejamento do desenvolvimento cultural. Na verdade, sua criação está diretamente ligada à formação e pesquisa (OEI, 2002).

Como incentivo ao estabelecimento dos Observatórios desse tipo, pode-se mencionar a iniciativa da UNESCO, ao estabelecer a Rede Internacional de Observatório das Políticas Culturais, com o objetivo de promover a conexão através de Internet entre entidades do mundo todo que analisam e reúnem e difundem conhecimento e informação que dizem respeito à Política Cultural e que tem como objetivos o estabelecimento de interações internacionais sistemáticas para o intercâmbio de informação, melhoria da base de conhecimentos para desenho e avaliação de Políticas Culturais e promoção de estudos avaliativos orientando o futuro de políticas culturais (OEI, 2002).

A rede foi formada em 2000 e é composta por observatórios que sejam organizados pela sociedade civil, autarquias públicas ou centros de abrangência internacional. É financiada pela UNESCO e possui abrangência mundial (RUBIM, 2005).

Outras iniciativas para estímulo a criação ou fortalecimento de observatórios foram mencionadas por Herschmann (2006). O autor referia-se mais especificamente a observatórios de informação, comunicação e cultura. Foram elas: o II Fórum Social Mundial, ocorrido em Porto 
Alegre no ano de 2002, onde foi promovido o lançamento do Observatório Internacional de Meios de Comunicação; a Oficina de Trabalho Observatórios e ouvidorias cidadãs dos meios de comunicação na América Latina, ocorrida em Buenos Aires no ano de 2003; o /l Encontro Internacional sobre Diversidade Cultural - As Indústrias Culturais na Globalização, também ocorrido em Buenos Aires, no ano de 2004, que contou com uma mesa-redonda reunindo especialistas, intitulada Observatórios de políticas culturais: experiências locais e regionais (HERSCHMANN, 2006).

Outra ação teve bastante relevância, por evidenciar a importância da cultura e de instituições de planejamento e sistematização de dados, abrangendo inclusive regiões supranacionais. Em 2012, em Salamanca - Espanha, na XV Conferência Ibero-americana de Cultura, por iniciativa da Organização dos Estados Ibero-americanos (OEI), formou-se um grande observatório que abarca toda a região da ibero-américa. A intenção é realizar um projeto de cooperação cultural que buscará reunir e difundir informação sobre cultura e cooperação cultural, além de contribuir para elaboração de uma metodologia de indicadores culturais de interesse comum para a região (OIBC, 2013).

No caso da América Latina, Octavio Getino (2007) aponta a influência de aspectos econômicos e do neoliberalismo na instauração dos observatórios de cultura. Segundo o autor, a instalação de políticas neoliberais nos anos 90 fazem alguns governos adotarem medidas para conhecer a dimensão econômica e social da cultura e, em particular, das indústrias culturais. "essas iniciativas governamentais de alguns países latino-americanos inauguraram na região um grupo de trabalho empreendido por organizações governamentais europeias e de outras regiões, alguns anos antes".
Entre as iniciativas positivas da região, segundo organismos internacionais e nacionais, incluem-se os esforços para a criação de observatórios culturais que, em sua maioria, orientam-se para o tratamento das políticas culturais. Em termos gerais eles começaram a trabalhar em algumas universidades, talvez com a idéia de que os espaços acadêmicos pudessem obter e colocar em prática informações e estudos mais confiáveis do que aqueles que pudessem surgir de organizações governamentais. (GETINO, 2007, p. 47).

A cultura tem assumido relevância, tal como outras áreas como educação e saúde. As políticas culturais, na era da democracia, têm sido reconhecidas como potenciais propulsores de desenvolvimento e cidadania. Assim,

a cultura constitui agora uma questão de relevo para todos. $\mathrm{Na}$ era das democracias, tornou-se mesmo um direito do cidadão e o acesso a ela sinaliza o desenvolvimento geral de uma sociedade. A capacidade de participar da cultura, de criá-la e de legá-la às gerações futuras transformou-se em um indicador das melhorias sociais e econômicas, um sinal de que a cada geração, o conjunto da sociedade está envolvido por uma dinâmica de progresso (TOLILA, 2007, p. 35).

Para formulação de políticas culturais que assumem essa responsabilidade, é preciso formulá-las adequadamente, conforme as demandas reais da sociedade. Para tanto, pesquisa, base de dados e indicadores culturais tornam-se instrumentos basilares. Esses fatores

permitirão, eventualmente, que se faça uma política cultural distinta daquela até hoje predominante e que se baseia em opiniões e crenças dos emissores de política cultural (entre 
eles, o maior deles, o Estado). Com o observatório, as políticas culturais podem eventualmente, tomar como referência o receptor das políticas culturais - que nesse ato se transforma, necessariamente, em emissor de cultura (COELHO, 2007, p. 11).

\subsection{Definição e tipologias dos Observatórios}

A primeira parte deste artigo foi dedicada ao contexto e às motivações para o surgimento dos Observatórios de Cultura e de Políticas Culturais. Neste subtópico serão explicitados os diferentes tipos de observatórios de cultura que foram criados, para um posterior esforço de categorização. Entretanto, antes de entrar nesta seara, é importante rever alguns esforços já feitos no sentido de dar corpo a uma definição para este tipo de instituição.

Afinal, o que são e qual seria o papel dos observatórios? De modo geral entende-se que "um Observatório, qualquer que seja o desenho institucional escolhido para seu funcionamento, deverá coletar, organizar, sistematizar, tornar compreensíveis e difundir informações objetivas sobre a cultura e reflexões sobre valores culturais (OBSERVATÓRIO ITAÚ CULTURAL, 2007, p. 22).

Paul Tolila (2007) ofereceu algumas contribuições a respeito da criação de observatórios ${ }^{2}$. O autor defende que um observatório deve ser um instrumento permanente, que atue de modo contínuo e disponha de recursos, além de uma organização adaptada. "Ao fomentar o conhecimento dos fenômenos culturais, ele contribuirá para desenvolver políticas públicas e enriquecerá o debate aberto sobre as questões de cultura. O observatório deverá formular, por meio de suas atividades, projetos de cooperação no país e no exterior" (TOLILA, 2007:35).
$\mathrm{Na}$ opinião do autor, um observatório não é um simples laboratório acadêmico em que a pesquisa é guiada unicamente por preocupações intelectuais. Tal como num laboratório, metodologias e critérios científicos seriam acionados para almejar a credibilidade e a eficácia, assim como disciplinas como economia, estatísticas e sociologia. Mas, o observatório estaria a serviço das políticas públicas e as perguntas que orientariam suas pesquisas e ações estariam fora dele: seriam orientadas pelas estratégias dos gestores públicos, pelos eixos determinantes das políticas culturais e pelas demandas do debate público.

Entretanto, de acordo com o autor, o observatório não deve ser encarado como órgão de planejamento ou de formulação estratégica. Seus trabalhos devem contribuir para planejamento e elaboração de estratégias, mas sem se transformar em arena de decisão política. Um observatório que sirva apenas de instrumento do poder, ou seja, para legitimar políticas realizadas sem qualquer análise crítica, perderá sua credibilidade e confiabilidade. Nesse caso ele apenas será uma forma disfarçada de publicidade e sua produção deixa de ser respeitada.

Por último, Paul Tolila (2007) também frisa a importância da continuidade nos estudos, a fim de produzir conhecimentos válidos para apontamentos para elaboração de políticas púbicas.

Apesar das contribuições mencionadas acima, o vultoso crescimento de observatórios, nos mais diversos formatos e propósitos, torna a definição algo mais complexo. Os observatórios de maneira geral, para além daqueles cuja temática é cultura-política cultural, podem ser divididos em dois grandes conjuntos de acordo com as funções que assuem: ou são espaços articuladores de cidadania e fiscalização, para monitoramento - observatório fiscal -; ou são espaços de colaboração para formu- 
lação de políticas públicas - observatório thinktank. (HERSHMANN, 2006).

O primeiro tipo estaria fortemente vinculado aos conceitos de cidadania e democracia participativa. Funcionaria como uma janela para o cidadão comum observar as políticas púbicas e acessar a análise crítica produzida. É um canal importante para o exercício do controle social, ou seja, é um fomentador para mecanismos de accountability. São exemplos os observatórios da rede de Observatórios Sociais do Brasil (SCHOMER e MORAES, 2010).

Já nos observatórios thinktank o trabalho de observação e análise não é somente o de crítica, mas também o de orientação, ideias e propostas para o aperfeiçoamento das políticas de algum setor, sempre tendo como parâmetro os interesses coletivos e não somente os interesses de um setor específico (GETINO apud HERSCHMANN, 2006). A este tipo de observatório cabem articular pesquisas, diagnósticos, indicadores e avaliações com a elaboração de políticas públicas. Comumente este observatório pertence à estrutura do Estado, sendo, portanto financiado por ele.

Apesar dessas contribuições para categorização dos observatórios, quando se trata de cultura e políticas culturais é possível ver uma multiplicidade de modelos surgindo, sejam eles subcategorias desses dois grandes conjuntos, ou ainda formatos que não são contemplados por eles. Alguns nascem com propósitos muito mais acadêmicos e inclusive estão vinculados à universidades. Outros têm suas ações mais voltadas para divulgação de ações culturais e seus efeitos. Alguns ainda, para além da prática da pesquisa e da construção de indicadores, organizam intervenções artísticas e culturais.

$\mathrm{Na}$ próxima sessão deste artigo busca-se dar mais embasamento à análise dos formatos de alguns observatórios existentes na América Latina.

\subsection{Levantamento de Obser- vatórios}

Para esta pesquisa exploratória foram levantadas informações de 20 (vinte) Observatórios com temática relacionada à Cultura e/ou Políticas Culturais que foram criados em quatro países da América Latina: Brasil, Argentina, Chile e Colômbia, estando estes atuantes ou não. Buscaram-se informações julgadas cruciais para contribuir na construção de tipologias, tais como: a missão da instituição, as fontes de financiamento, principais ações. Aintenção é verificar nas experiências já concretizadas, inspiração para desenhar categorias de observatórios.

Por se tratar de uma pesquisa exploratória, o meio de acesso às informações utilizado foram os canais oficiais divulgação dos observatórios, seus websites. Para elaboração de tipologias - ou tipos ideais - as declarações advindas em discursos oficiais dos próprios observatórios, mostraram-se suficientes. Em momentos posteriores, a intenção é abarcar outras fontes para verificar as ações dos mesmos.

Além disso, os websites enquanto canais de divulgação foram escolhidos a partir do pressuposto da acessibilidade. Um observatório deve zelar pela publicização das informações, pois

os resultados de sua investigação devem ser apresentados na forma de produtos de acesso público garantido (...) A comunicação e a difusão de informações devem ser entendidas como esferas estratégicas de um observatório voltado para a atuação em redes de intercâmbio e de cooperação e que advogue para si um papel de protagonista no campo das artes e da cultura. Trata-se de elaborar e distribuir sistematicamente produtos como um site, boletins eletrônicos, boletins impressos, publicações em papel que comentem e apresentem as pesquisas 
efetuadas, revistas e outros periódicos impressos ou eletrônicos, livros que condensem resultados de simpósios e seminários (OBSERVATÓRIO ITAÚ CULTURAL, 2007, p. 23).

Assim sendo, assumindo a internet como um canal ótimo de divulgação de seu funcionamento, neste primeiro momento da pesquisa, a coleta de dados utilizou os websites institucionais como principal fonte.

\subsection{Propostas de tipologias}

A pesquisa preliminar visou identificar alguns dos Observatórios que foram criados nos quatro países já mencionados. Nestes, objetivou-se identificar as informações sobre missão, vínculo institucional, histórico, principais ações desenvolvidas, dentre outras disponíveis nos sítios institucionais destes organismos.

A escolha destes como ponto de partida nesta pesquisa se deu pelo fato de constituírem uma rica amostragem, uma vez que incluem a diversidade nas variáveis ${ }^{3}$ que se pretende observar: diferentes tipos de vínculos institucionais (acadêmico, poder público, sociedade civil e entidade privada); diferentes missões e ações (fornecer dados para indústria cultural; fomentar e sugerir políticas culturais; fiscalizar ações do poder público; gerar redes; reunir e fomentar pesquisas acadêmicas; formar gestores e agentes culturais; publicações etc.); diferentes contextos e raio de atuação (municipal, regional, nacional, supranacional).

Embora na prática as missões e ações se entranham de forma mais complexa que na formulação de tipos ideais, com um esforço de reflexão, foi possível delinear três grandes categorias:

$1^{a}$. Aqueles vinculados a algum órgão do poder público e dedicados ao fomento de política pública; $2^{a}$. Aqueles vinculados a órgãos acadêmicos priorizando pesquisas acadêmicas e divulgação no meio científico;

$3^{a}$. Aqueles vinculados à sociedade civil ou iniciativa privada com atividades mistas.

CATEGORIA 1: vinculados a algum órgão do poder público e dedicados ao fomento de política pública

A maior parte dos Observatórios que compuseram o universo da pesquisa nasce vinculada ao setor cultural do poder público, financiado pelo poder público, com o propósito de levantar dados e mapear demandas para fomentar a formulação de Políticas Culturais, bem como, fazer estudo de impacto de ações e políticas culturais. Em geral, mantêm boas relações com a sociedade civil e instituições acadêmicas. Muitos geram anuários e boletins estatísticos.

- Observatorio de Industrias Creativas (Buenos Aires - Argentina)

Constitui um Sistema de informações quantitativas e qualitativas voltado para indústrias culturais, para orientar ações tanto do poder público, quanto do setor privado.

- Observatorio Cultural com Orientación Bibliotecológica y Promoción de la Lectura (Buenos Aires - Argentina)

É vinculado ao Setor de Bibliotecas do governo da cidade de Buenos Aires e visa divulgar condutas e práticas culturais para o fomento de políticas públicas para a promoção de leitura. Mantém um serviço de divulgação: de endereço e horário de funcionamento das bibliotecas, de artigos sobre a importância da leitura, dos censos das bibliotecas, de publicações e congressos sobre o tema e um boletim cultural. 
- Observatório da Cultura de Porto Alegre (Rio Grande do Sul - Brasil)

Além da pesquisa, traça vínculos importantes com a sociedade civil, como Conselho Municipal de Cultura e Universidade. Promove audiências públicas. Realiza programas de formação em cultura e assessora o poder público na implantação do Sistema Municipal de Cultura e dos elementos que o constituem.

\section{- Observatório Brasileiro de Eco- nomia Criativa (Brasil)}

Foi criado pelo Ministério da Cultura e mantém uma relação de parceria com algumas Universidades Federais. É uma instância responsável pela produção e difusão de pesquisas, dados e informações sobre economia criativa no país, assim como pelo estímulo ao debate entre estudiosos, especialistas, agentes governamentais e representantes do setor cultural sobre os impactos da Economia Criativa na sociedade. Visa produção de conhecimento para o fomento de política pública direcionada à economia criativa.

\section{- Observatório Afrolatino (Brasil)}

Fundado em 2009, é de responsabilidade do Centro Nacional de Informação e Referência da Cultura Negra - CNIRC, órgão da Fundação Cultural Zumbi dos Palmares. Apesar de ser vinculado a um órgão público pertencente ao Ministério da Cultura, este observatório se adéqua melhor na segunda categoria, pois suas atividades estão mais relacionadas à divulgação de pesquisas. Trata-se de um programa colaborativo para conexões, intercâmbios e diálogos das culturas afro-latinas e caribenhas. A intenção é conhecer, reunir, e divulgar informações sobre as diferentes culturas de raízes negras latinas e caribenhas. O observatório aceita o envio de qualquer tipo de estudo, os organiza em eixos temáticos, juntamente com outras informações como legislação, artigos, livros, revistas e fotos.
- Observatório Cultural del Departamento de Estúdios del Consejo de la Cultura y las Artes (Chile)

Além da produção de dados e pesquisa para o fomento de política pública (linhas: comunidade, território e patrimônio; consumo e participação cultural; economia da cultura e gestão cultural), possui publicação periódica de revista. Focos: estudo, reflexão e pesquisa.

\section{- Observatório de Culturas (Bo- gotá - Colômbia)}

Anteriormente conhecido como Observatorio de Cultura Urbana del Instituto Distrital de Cultura y Turismo, possui forte atuação no campo da pesquisa, para formulação e desenho de políticas públicas. Mede e registra as ações da Secretaria de Cultura, Recreación e Deporte, tais como grau de satisfação, características e quantidade de público. Publica anuários e realiza a "Encuesta Bienal de Culturas", uma ferramenta - transformada numa publicação periódica - com análise da situação cultural de Bogotá para orientar política pública.

- Observatorio de Patrimonio Cultural MIA (Instituto Colombiano de Antropología e Historia - Ministério de Cultura - Colômbia)

Trata-se do setor de pesquisa do instituto. $O$ foco é a pesquisa e a reflexão teórica. Analisa processos sociais, políticos e econômicos da instrumentalização do discurso do patrimônio. Desenvolve conceitos acadêmicos e cria espaços de discussão sobre políticas culturais relativas ao patrimônio. Produziu reflexões para estabelecimento de marcos nas políticas públicas acerca de patrimônio.

\section{- Observatório Brasileiro do Cine- ma e do Audiovisual - ANCINE (Brasil) \\ Vinculado â Agência Nacional do Cinema, visa produzir dados para fomen-}


tar políticas públicas para o setor audiovisual. Elabora e divulga estudos estatísticos detalhados sobre salas de cinema, TV paga, produção, arrecadação, informes, dentre outros.

\section{- Observatório dos Museus e Centros Culturais}

Criado pela Fiocruz, possui parceria com Museu da Vida, Casa Oswaldo Cruz, Diretoria Regional de Brasília da Fundação Oswaldo Cruz, Departamento de Museus do IPHAN. Sua missão é compor uma tecnologia de gestão e construção de informações e conhecimento sobre museus e suas relações com a sociedade, desenvolvida em parceria com instituições de pesquisa e gestão pública; e ser um programa de serviços e pesquisa, voltado para ampliação da reflexão sobre museus, visando subsidiar políticas públicas e apoiar gestão das instituições museais. As ações envolveram a realização de pesquisas, bases de dados, capacitação e divulgação de produtos para gestores e formuladores de políticas públicas para o setor de museus.

CATEGORIA 2: Aqueles vinculados a órgãos acadêmicos priorizando pesquisas acadêmicas e divulgação no meio científico

Os observatórios diretamente vinculados às Universidades compuseram grande parte dos observatórios pesquisados. Alguns, embora ambicionem mais, parecem se constituir apenas como um repositório e divulgação de pesquisas acadêmicas no campo da cultura; outros, para além do campo da pesquisa, buscam fiscalizar, influenciar e propor políticas culturais de suas regiões. De todo modo, todos possuem um papel chave na formação de redes de envolvidos com cultura e políticas culturais, e na grande maioria, oferecem cursos de capacitação e seminários e até mesmo assessorias/consultorias.
- Observatório Cultural de la Universidad Nacional de Formosa (Argentina)

Objetiva reunir, processar e difundir informação relativa ao movimento econômico, político e social do setor cultural. Essas informações serviriam de insumo e incentivo para o desenvolvimento de bens e serviços culturais na província de Formosa. Propõese a fazer levantamentos de dados sobre o setor cultural em outras áreas, como educação, democracia e diversidade cultural, para perceber o impacto das políticas culturais a nível local, regional e internacional.

- Observatorio Cultural de la Facultad de Ciencias Económicas de la Universidad de Buenos Aires (Argentina)

Propõe-se a promover pesquisas e programas de formação, assistência técnica, contribuindo assim para profissionalização da administração e gestão do setor cultural e criativo.

- Observatorio de Ciudadania Cultural Universidad Nacional de Avellaneda (Argentina)

Propõe-se a observar, descrever e analisar as diversas manifestações culturais, das expressões culturais da cidadania e das relações geradas entre os diversos aparatos governamentais com a sociedade civil. Com atividades estritamente acadêmicas, seus projetos são de pesquisa e extensão, mas não de fomento à política pública. Possui grande número de parcerias com outras universidades.

- Observatório Cultural UnilasaIle (Universidade Unilasalle - Canoas/ RS - Brasil)

Núcleo de Pesquisa e Extensão da Universidade, vinculado a um programa de Pós-Graduação. Realiza oficinas, divulga publicações acadêmicas e eventos e produz eventos acadêmicos e artísticos. 
- Observatório de Políticas Culturais (Universidade Federal da Paraíba - Brasil)

Trata-se de um grupo de pesquisa e extensão, que se propõe a produzir e difundir conhecimento sobre política e gestão cultural, a monitorar o desenvolvimento de ações neste campo, assim como gerar indicadores e informações que subsidiem as gestões públicas ligadas ao setor.

\section{- Observatório de Políticas e} Práticas Culturais e Birô Cariri (Ceará - Brasil)

Foi criado através de uma parceria entre Universidade Federal do Cariri e o SESC de Juazeiro do Norte. Apesar de ter o SESC como figura importante em sua constituição, este observatório é melhor posto na segunda categoria, já que se propõe principalmente o fortalecimento da pesquisa sobre os fenômenos culturais do Cariri Cearense. Seu foco recai sobre cultura e economia da cultura. O Birô, órgão que nasce vinculado ao observatório ficará mais responsável por promover ações culturais de qualificação ou intervenção.

\section{- Observatorio de Políticas Cul- turais (Universidad de Chile - Chile)}

Vinculado à Universidad de Chile, com apoio da Embaixada da França e da Sociedade Chilena do Direito do Autor e patrocinado pela UNESCO, o observatório visa promover estudo e pesquisa no setor cultural. Realiza assessorias em gestão cultural e formação em gestão cultural. Divulga suas pesquisas, leis, ações de políticas públicas e notícias relacionadas às políticas culturais. Divulga estudos de outras instituições. Na assessoria, o observatório vai até as cidades para montagem de um plano municipal de cultura, com instâncias participativas, agentes culturais e encontros com a comunidade. Depois, pode ainda, atuar na implantação deste plano.
CATEGORIA 3: Aqueles vinculados à sociedade civil ou iniciativa privada com atividades mistas

Incluem-se nesta categoria observatórios mantidos por organizações da sociedade civil ou da iniciativa privada. Eles fogem às características gerais apontadas nas categorias 1 e 2, mas divergem entre si, e assim essa tipologia ainda precisa ser melhor desenhada. Em outras áreas, observatórios mantidos pela sociedade civil costumam exercer função de promoção de cidadania e controle social do poder público. Mas, nos observatórios de cultura pesquisados não foi encontrada essa característica. Aqui eles continuam propondo reflexões acadêmicas e iniciativas de formação.

\section{- Observatório Iberoamericano de Cultura y Desarrollo Social (Argentina)}

Uma reunião de várias organizações da sociedade civil, pretende viabilizar canais sistemáticos de coleta, análise e intercâmbio de saberes e experiências entre indivíduos, grupos, ONGs, artistas, instituições públicas e privadas, que trabalhem com cultura aplicada ao desenvolvimento social. Já organizou jornadas, capacitações e publicações sobre artes.

- Observatório da Diversidade Cultural (Minas Gerais - Brasil)

É uma organização não governamental. Propõe-se a desenvolver programas de ação colaborativa entre gestores culturais, artistas, arte educadores, agentes culturais e pesquisadores. Objetiva produzir, informações e conhecimento, atuando sobre os desafios da proteção e promoção da diversidade cultural. Estimula o campo acadêmico e a principal atuação é em promoção de seminários, debates e cursos de formação. Produz publicações de livros e boletim periódico e realiza consultorias. 


\section{- Observatório Itaú Cultural (São Paulo - Brasil)}

O observatório é um projeto dentro do Instituto Itaú Cultural, este, voltado para a pesquisa e a produção de conteúdo e para o mapeamento, o incentivo e a difusão de manifestações artístico-intelectuais. O setor do observatório visa estudar e debater gestão, economia e políticas culturais, estimular a reflexão sobre eles e analisar os indicadores nacionais. Fomenta a pesquisa em cultura, com apoio a grupos de pesquisa e publicações como a Revista Observatório e livros, e o programa Rumos Itaú Cultural Pesquisa ${ }^{4}$. Fornece um curso de especialização em gestão cultural em parceria com a UNESCO e Universidade de Girona e com apoio da OEl. O maior foco é o fomento de pesquisa de órgãos externos. $O$ instituto fomenta atividades artísticas entre outras; porém, estas não ficam a cargo do setor do Observatório.

\subsection{Considerações Finais}

Em vias das considerações finais, ressalta-se a importância dos observatórios na sociedade atual, onde a cultura assume papel de grande relevância para cidadania, constituição de identidade e motor de desenvolvimento. Numa democracia, é papel do poder público pautarse em demandas reais para formular políticas públicas de interesse coletivo. Para tanto ouvir demandas, identificar os gargalos, propor indicadores culturais, pesquisar a evolução de comportamentos é algo que se faz fundamental.

O consenso sobre a necessidade de pesquisa criteriosa e reflexão sobre o tema é um grande impulsionador para o surgimento cada vez mais crescente dos observatórios. O fenômeno é tão gritante e ao mesmo tempo tão veloz que as pesquisas que buscam essa au- torreflexão são escassas e rapidamente tornam-se obsoletas.

Este pequeno artigo tem a intenção de chamar atenção para a necessidade de identificar e analisar o surgimento e atuação desses observatórios. A proposta de três tipologias que aqui se fez, não deve ser inflexível. É, na verdade, um pontapé inicial para reflexão por parte de organizações que almejem tornar-se observatórios (ou para os observatórios já existentes), para que reflitam sobre seu papel social e definam com clareza e planejamento sua missão, propostas de ações, raio de alcance, parceiros, dentre outros elementos que irão influenciar profundamente o ser e o fazer da instituição. O artigo transformase também num convite para que outros pesquisadores da área de políticas culturais ingressem na temática e auxiliem no desvelar de questões ainda obscuras ou até mesmo ainda não formuladas.

Afinal, fica cada vez mais clara a capacidade e a relevância que os observatórios têm para: pesquisa, estabelecimento de indicadores e avaliação e proposição de políticas públicas. Fica também claro o papel que eles exercem na circulação da informação e no intercâmbio de experiências, bem como na qualificação e profissionalização para o campo da gestão cultural. Estes fatores sem dúvida transformam comportamentos da sociedade; e por isso, a reflexão se faz urgente.

Para concluir, é válido frisar que em países com tristes tradições, como por exemplo o Brasil, os observatórios possuem o poder de vincular as políticas culturais à demanda da sociedade e não à grupos privilegiados, ou à opinião particular de um gestor, conforme era (e ainda é) tradicionalmente feito. É por isso que uma definição madura na construção desses organismos tende a favorecer a sociedade como um todo. 


\section{Bibliografia}

ALBORNOZ, Luís; HERSCHMANN, Micael. Os observatórios ibero-americanos de informação, comunicação e cultura: balanço de uma breve trajetória. Revista da Associação Nacional dos Programas de Pós-Graduação em Comunicação. Dezembro de 2006.

COELHO, Teixeira. Da Opinião ao Dado. Revista Observatório Itaú Cultural/OIC - n. 1 (jan./abr. 2007) - São Paulo: Itaú Cultural, 2007.

GETINO, Octávio. Experiências de alguns observatórios Culturais: para a melhoria das políticas e desenvolvimento do setor cultural. Revista Observatório Itaú Cultural/OIC - n. 1 (jan./abr. 2007) São Paulo: Itaú Cultural, 2007.

OBSERVATÓRIO ITAÚ CULTURAL. A experiência Internacional e a Criação do Observatório Itaú Cultural. Revista Observatório Itaú Cultural/ OIC - n. 1 (jan./abr. 2007) - São Paulo: Itaú Cultural, 2007.

OEI - Organização dos Estados Ibero-americanos. Las políticas y laslegislacionesculturales. In: Agenda Iberoamericana de la Cultura. Madrid, 2002. Disponível em http:// www.oei.es/agendacultural/inicio.htm, acessado em 02/01/2015.

OIBC - Observatório Iberoamericano de Cultura. Boletín n. 0. 21 de maio de 2013. Disponível em: www.oibcult.org/web/?BOLETIN-NO-1, acessado em 15/01/2015.

ORTEGA, Cristina; VALLE, Roberto San Salvador del. Nuevos retos de los observatórios culturales. Boletín Gestión Cultural. N. 19: Observatórios Culturales. Enero de 2010.

RUBIM, Antônio Albino Canelas; PITOMBO, Mariella; RUBIM, luri Oliveira. Políticas e Redes de Intercâmbio e Cooperação em Cultura no Âmbito Ibero-Americano. In: CONVÊNIO ANDRÉS BELLO. Siete cátedras para la integración. Bogotá, CAB, 2005, p. 129-170. Disponível em: http:// www.cult.ufba.br/arquivos/cult_politicas_e_redes. pdf, acessado em 10/01/2015.

SCHOMMER, Paula Chies; MORAES, Rubens Lima. Observatórios sociais como promotores de controle social e accontability: Reflexões a partir da experiência do Observatório Sociais de Itajaí. Gestão.Org Revista Eletrônica de Gestão Organizacional - 8 (3): 298-326 Set/Dez 2010.
TOLILA, Paul. Observatório Cultural: Ferramenta democrática de ação política. Revista Observatório Itaú Cultural/OIC - n. 1 (jan./abr. 2007) - São Paulo: Itaú Cultural, 2007.

\section{Recebido em 18/01/2015 Aprovado em 23/02/2015}

1 Clarissa Alexandra Guajardo Semensato, Rio de Janeiro, Brasil. Licenciada em Geografia pelo Instituto Federal Fluminense de Campos (IFF- Campos), Bacharel em Ciências Sociais e Mestre em Políticas Sociais pela Universidade Estadual do Norte Fluminense Darcy Ribeiro. Atualmente é bolsista pesquisadora do Setor de Estudos em Políticas Culturais da Fundação Casa de Rui Barbosa (FCRB). Contato: clarissaalexandra@gmail.com

2 Nesta ocasião Paul Tolila escreve especificamente para o Brasil. Entretanto, suas orientações podem ser estendidas a outras realidades.

3 Para este artigo não serão expostos todo o volume de dados acumulado na busca por estas variáveis. Optou-se por um compilado das características principais a fim de compor as três tipologias sugeridas. Pretende-se utilizar este banco de dados futuramente, incorporando novos dados e novos observatórios.

4 Rumos Itaú Cultural Pesquisa: Gestão Cultural, que tem, entre seus objetivos, o propósito de promover a formação e o desenvolvimento dos profissionais e pesquisadores atuantes nas instituições culturais brasileiras. Assim, incentivam projetos de pesquisa acadêmica capazes de abranger questões relativas à gestão cultural, no âmbito das políticas culturais, dos processosde produção cultural, da economia da cultura ou da gestão de organizações públicas e privadas (OBSERVATÓRIO ITAÚ CULTURAL, 2007) 